\title{
InspSoft 2.0: Um Jogo para o Ensino de Inspeção de Software
}

\author{
Adriana Lopes ${ }^{1,2}$, Davi Viana ${ }^{1}$, Jacilane Rabelo ${ }^{1}$, Andréia Vieira ${ }^{2}$, Tayana Conte ${ }^{1}$ \\ ${ }^{1}$ USES - Grupo de Usabilidade e Engenharia de Software, Universidade Federal do \\ Amazonas (UFAM), Manaus - Amazonas - Brasil \\ ${ }^{2}$ FPF - Fundação Paulo Feitoza, Manaus - Amazonas - Brasil \\ \{adriana, davi.viana, jaci.rabelo, tayana\}@icomp.ufam.edu.br, \\ avieiradfpf.br
}

\begin{abstract}
Software Inspection is a formal process that includes a set of activities for guaranteeing the software quality. In order to execute such activities, it is necessary a specific knowledge and suitable professionals' qualification. One way to support better learning is through educational games. These games can facilitate the teaching-learning process, strengthening inspection concepts through practice. This paper presents the game InspSoft 2.0 that supports Software Inspection learning. Additionally, we describe results of its evaluations that allowed us to verify issues regarding learning through game.
\end{abstract}

Resumo. Inspeção de Software é um processo formal que possui um conjunto de atividades visando garantir a qualidade dos artefatos de software. Para executar tais atividades, são necessários conhecimentos especificos e uma adequada capacitação de profissionais. Uma forma de auxiliar na melhor aprendizagem é através de jogos educacionais. Esses jogos podem facilitar o processo de ensino-aprendizagem, reforçando conceitos através da prática. Desta forma, este artigo apresenta o jogo InspSoft 2.0 que apoia na aprendizagem de Inspeção de Software. Adicionalmente, são descritos os resultados de avaliações que permitiram verificar questões relacionadas ao aprendizado através do jogo.

\section{Introdução}

A Qualidade de Software tem por objetivo garantir a conformidade de processos e artefatos produzidos, eliminando os defeitos que são encontrados em todas as fases do processo de desenvolvimento [Bartié, 2002]. Segundo Bertini et al. (2006), 60\% a 90\% dos defeitos podem ser encontrados na fase inicial do processo de desenvolvimento. Porém, se técnicas adequadas não forem aplicadas, estes defeitos são detectados apenas nas fases finais, acarretando prejuízos, pois o custo para corrigir um erro cresce com o tempo [Kalinowski et al., 2004]. Desta forma, torna-se necessário um processo de Garantia da Qualidade que atue em todas as fases do processo de desenvolvimento [Magalhães et al., 2008].

Inspeção é uma técnica de revisão formal de software utilizada em diversas fases do processo de desenvolvimento para descobrir antecipadamente defeitos e corrigi-los a 
um custo menor e de forma eficiente [Kalinowski et al., 2004]. Além disso, técnicas de revisão formal são requeridas pelo processo de Verificação do MR-MPS (Modelo de Referência de Melhoria do Processo de Software) [Softex, 2011] e pela área de processo equivalente do CMMI (Capability Maturity Model Integration) [SEI, 2010]. Deste modo, observa-se que a realização de inspeção de software é uma prática importante nas organizações.

No entanto, se as atividades de inspeção de software não forem feitas de forma adequada, elas podem prejudicar seu verdadeiro potencial [Ciolkowski et al., 2003]. Capacitar profissionais e estudantes é uma das alternativas a serem usadas para possibilitar o uso mais eficiente de inspeções pela indústria de software. Porém, um dos problemas relacionados à capacitação de métodos e técnicas de Engenharia de Software (ES) se deve ao fato de que as iniciativas de treinamento muitas vezes possuem um enfoque teórico [Thiry et al., 2010].

Uma forma de estimular o aprendizado e diminuir o enfoque teórico é através do uso de jogos educacionais. Eles podem tornar o processo de ensino-aprendizagem mais atrativo e proveitoso [Kochanski et al., 2009], permitindo reforçar conceitos através da prática [Monsalve et al., 2010]. Segundo Alexander e Beatty (2008), a utilização de jogos aumenta o envolvimento emocional de um estudante, ocasionando variações de estímulos, ajudando-o a reter novos conhecimentos. Com o objetivo de auxiliar na retenção de conhecimentos relacionados à inspeção de software, desenvolveu-se o jogo educacional InspSoft [Lopes et al., 2012]. De forma lúdica, este jogo proporciona aprendizado ao processo de inspeção, com ênfase na atividade de detecção de defeitos e papéis dos participantes.

Visando melhorias no ambiente do jogo InspSoft, este artigo apresenta as evoluções do jogo feitas a partir de um estudo preliminar. Além disso, realizou-se uma nova avaliação do jogo. As próximas seções deste artigo estão organizadas da seguinte forma: a Seção 2 apresenta os principais conceitos de inspeção de software e jogos educacionais propostos para o ensino de inspeção de software. A Seção 3 descreve o jogo InspSoft 2.0, suas principais características e funcionalidades. A Seção 4 apresenta a avaliação do jogo através de um modelo para verificar a efetividade da aprendizagem. Por fim, a Seção 5 descreve as considerações finais e trabalhos futuros.

\section{Inspeção de Software e Jogos para Apoio à Aprendizagem}

A inspeção de software contém atividades que contribuem na melhoria da qualidade do software e nos ganhos significativos de prazos e custos [Silva et al., 2004]. Para a capacitação de profissionais qualificados na inspeção, os jogos educacionais podem ser utilizados como ferramenta de apoio ao ensino da inspeção de software. Nesta seção serão abordados o processo de inspeção e jogos educacionais para o apoio à aprendizagem de inspeção.

\subsection{Processo de Inspeção}

A inspeção é composta por um conjunto de atividades realizadas por uma equipe tecnicamente qualificada para verificar se o artefato possui qualidade satisfatória. Desde que o processo tradicional de inspeção de software foi definido por Fagan (1976), diversas variações sobre esse processo foram propostas, com o objetivo de aumentar a 
eficiência da inspeção [Kalinowski et al., 2004]. As atividades do processo de inspeção baseados na reorganização proposta por Sauer et al. (2001) são descritas na Tabela 1.

A atividade de detecção de defeitos pode ser realizada de modo Ad-hoc, ou através de uma técnica de apoio à inspeção [Bertini et al., 2006]. Dentre as técnicas de inspeção pode ser citada o Checklist, composto por uma lista de questões que orientam o inspetor na detecção de defeitos em um determinado artefato de software [Kalinowski et al., 2004]. Na literatura, existem outras técnicas de apoio a inspeção mais específicas, como a técnica baseada em perspectiva (PBR) para a inspeção em documentos de requisitos e a inspeção de projetos orientado a objetos (OORT's) [Mafra et al., 2006].

Tabela 1. Atividades do Processo de Inspeção

\begin{tabular}{|l|l|}
\hline Planejamento & $\begin{array}{l}\text { Atividade onde é feita a definição do escopo da inspeção, preparação do material } \\
\text { para a inspeção e seleção dos inspetores. }\end{array}$ \\
\hline Detecção & $\begin{array}{l}\text { Cada inspetor executa individualmente essa atividade, a qual consiste na busca } \\
\text { de possiveis defeitos (discrepâncias). }\end{array}$ \\
\hline Coleção & $\begin{array}{l}\text { Eliminação de discrepâncias repetidas (encontradas por mais de um inspetor), } \\
\text { gerando uma lista de discrepâncias únicas. }\end{array}$ \\
\hline Discriminação & $\begin{array}{l}\text { Classificação das discrepâncias em defeitos reais. As discrepâncias não } \\
\text { classificadas como defeitos são consideradas falso-positivos. }\end{array}$ \\
\hline
\end{tabular}

\subsection{Jogos de Apoio ao Aprendizado de Inspeção}

No ensino de conceitos relacionados à ES, é necessária uma interação entre aspectos teóricos e práticos, porém nem sempre os conceitos são cobertos satisfatoriamente com as práticas. Desta forma, torna-se relevante o uso de jogos com o objetivo de aumentar a cobertura dos conceitos e de exercitar situações nos processos de desenvolvimento de software [Monsalve et al., 2010].

A maioria dos jogos educacionais desenvolvidos para ES possui o foco do aprendizado voltado para o ensino de Gerência de Projetos ou para a ES de modo geral, onde surge a demanda por jogos educacionais que apoiem a aprendizagem em processos específicos na ES [Thiry et al. 2010]. Entre os jogos voltados para a aprendizagem de ES, foi identificado o jogo InspectorX [Pötter e Schots, 2011] como apoio ao ensino de inspeção em artefatos de software. O InspectorX disponibiliza questões com trechos de documentos de requisitos e código com um ou mais defeitos. A tarefa do jogador é selecionar o trecho que contenha o defeito e classificá-lo a partir das taxonomias de defeitos cadastradas no jogo.

Para apoiar e ampliar a aprendizagem de outros aspectos do processo de inspeção, um novo jogo foi proposto: o InspSoft. Entre outros aspectos, pode-se destacar o ensino de papéis dos participantes no processo de inspeção de software e atividade de detecção de defeitos em um artefato de software. Para verificar questões relacionadas à aprendizagem dos conceitos de inspeção de software, realizou-se uma avaliação preliminar. Esta avaliação permitiu verificar indícios sobre o ganho de conhecimento dos participantes [Lopes et al., 2012]. Os resultados preliminares permitiram realizar uma evolução no jogo, gerando uma nova versão que é apresentada a seguir. 


\section{InspSoft 2.0}

O jogo InspSoft tem por objetivo proporcionar o aprendizado ao processo de inspeção de software, papéis dos participantes em uma inspeção e na atividade de detecção de defeitos através de um ambiente lúdico, com a finalidade de entreter os jogadores através de seu enredo e dos personagens, simulando uma inspeção de software em uma empresa. Assim, é possível aprender sobre os papéis de cada participante no processo de inspeção e os tipos de defeitos existentes. A Tabela 2 apresenta a taxonomia dos defeitos de requisitos considerados neste trabalho.

A versão inicial do jogo InspSoft foi avaliada experimentalmente e publicada em Lopes et al. (2012). O InspSoft foi atualizado com o objetivo de melhorar o layout do jogo através de novas figuras dos avatares dos participantes no processo de inspeção e pelos tipos de defeitos considerados no jogo. Uma nova funcionalidade foi implementada para apoiar a inspeção em um artefato de software. Esse apoio é feito através de um Checklist, que pode ser utilizado em qualquer momento do terceiro nível do jogo. Nesta versão, também está disponível as respostas dos desafios deste terceiro nível, visto que foram consideradas as sugestões de melhoria dos participantes na avaliação preliminar da versão 1.0 do jogo. O InspSoft possui licença gratuita e está disponível em http://www.fpf.br/downloads/jogoInspecao_V2.rar.

Tabela 2. Tipos de Defeitos em Requisitos (adaptado de Travassos et al., 2001)

\begin{tabular}{|l|l|}
\hline Tipos de Defeitos & \multicolumn{1}{|c|}{ Descrição } \\
\hline Omissão & $\begin{array}{l}\text { Deve-se à omissão ou negligência de alguma informação necessária ao } \\
\text { desenvolvimento do software. }\end{array}$ \\
\hline Ambiguidade & $\begin{array}{l}\text { Ocorre quando uma determinada informação não é bem definida, permitindo } \\
\text { assim uma interpretação subjetiva, que pode levar a múltiplas interpretações. }\end{array}$ \\
\hline Fato Incorreto & $\begin{array}{l}\text { Informações dos artefatos do sistema que são contraditórias com o conhecimento } \\
\text { que se tem do domínio da aplicação. }\end{array}$ \\
\hline Inconsistência & Ocorre quando duas ou mais informações são contraditórias entre si. \\
\hline
\end{tabular}

\subsection{Visão Geral do Jogo}

A tela inicial do jogo apresenta todos os avatares utilizados no jogo e uma breve descrição sobre o InspSoft, após a opção "Jogar" ser selecionada, é apresentado um anúncio fictício de uma vaga de emprego para trabalhar em uma empresa de desenvolvimento de software, especificamente no grupo da Garantia de Qualidade. A Figura 1 apresenta essa tela, onde também é possível escolher o avatar para participar do jogo.

Após a escolha do avatar, o jogador desempenhará o papel do moderador e inspetor no processo de inspeção. Em seguida, o jogador é direcionado a tela com o cenário da sala do autor do Documento de Requisitos para receber o artefato a ser inspecionado, sendo sua "primeira atividade na empresa". Após o jogador selecionar o próximo cenário, o jogo disponibiliza três níveis de desafio onde cada um dos desafios devem ser realizados sequencialmente. No primeiro nível o jogador irá conhecer a equipe e deverá classificar o nome do papel dos participantes no processo de inspeção de acordo com a função. 


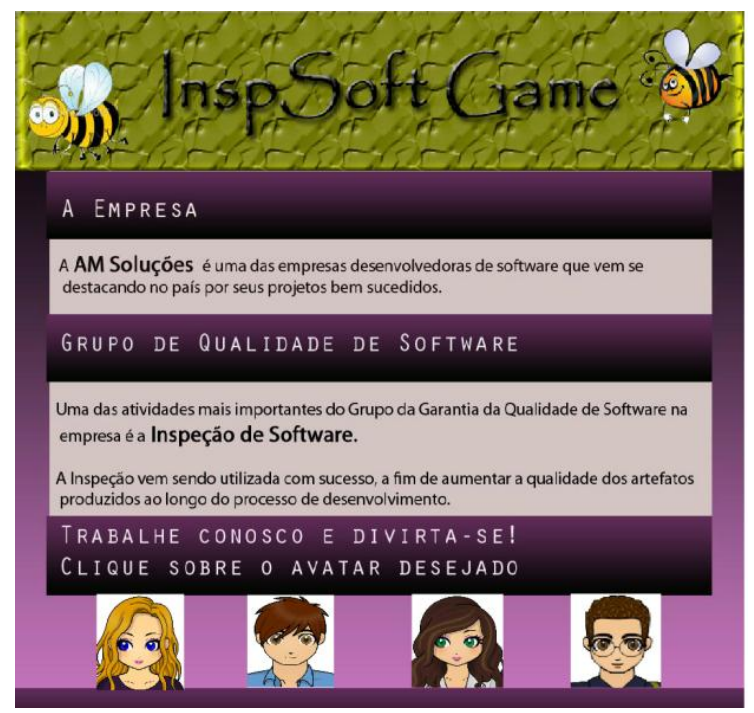

Figura 1. Cenário de anúncio da vaga e escolha do avatar.

O objetivo neste nível é adquirir conhecimento sobre os papéis de Autor, Moderador e Inspetor no processo de Inspeção de Software. A Figura 2.a apresenta a tela deste primeiro nível. No segundo nível, inicialmente, o jogador irá estudar os tipos de defeitos que podem ser encontrados no documento de requisitos. Em seguida, o jogo fornece exemplos de defeitos e solicita que o jogador classifique esses defeitos. A Figura 2.b. apresenta uma tela de classificação dos defeitos.

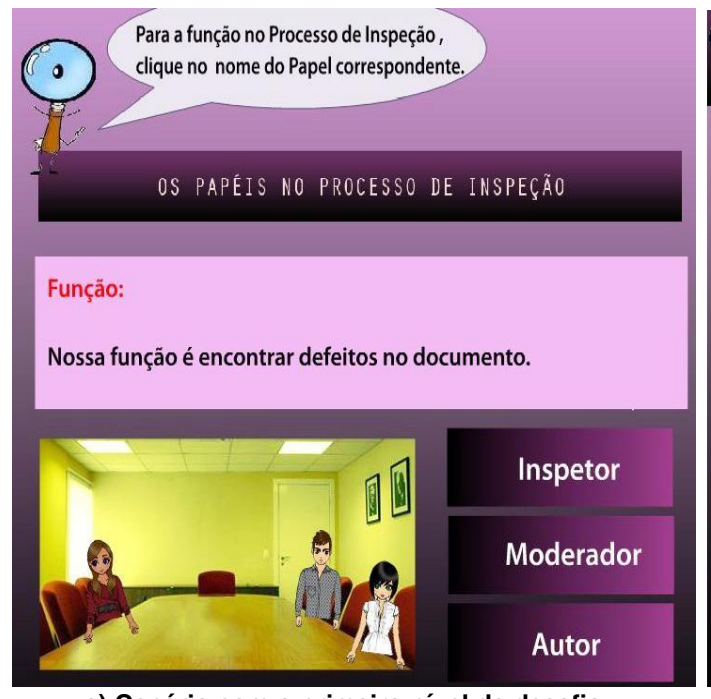

a) Cenário com o primeiro nível de desafio.

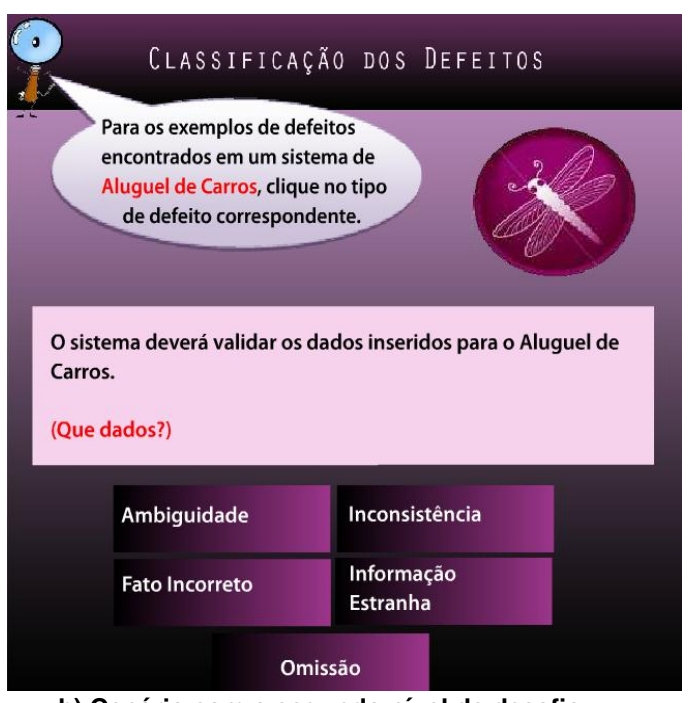

b) Cenário com o segundo nível de desafio. Figura 2. Desafios do Jogo - Primeiro e Segundo Nível.

No terceiro nível, é fornecida uma especificação de um caso de uso para o jogador analisar, conforme a Figura 3.a. Em seguida, o jogo disponibiliza trechos do caso de uso onde o jogador deve categorizar os tipos de defeito encontrados. Neste desafio, existe a opção que foi implementada para o jogador utilizar um Checklist como apoio à detecção de defeitos, como apresenta a Figura 3.b. 


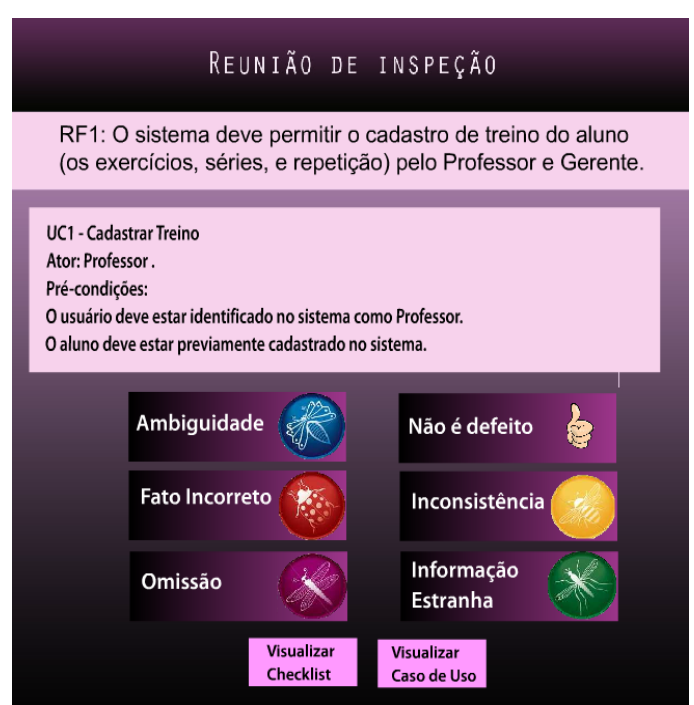

a) Cenário com o terceiro nível de desafio.

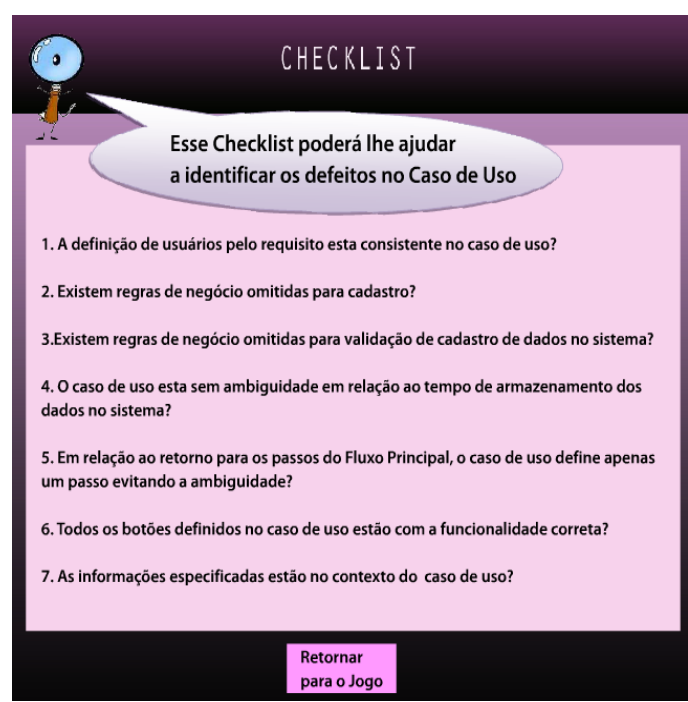

b) Checklist usado no terceiro nível de desafio.

Figura 3. Desafios do Jogo - Terceiro Nível.

Após o jogador concluir os desafios do terceiro nível, são disponibilizadas as respostas de cada item de desafio conforme a Figura 4.a. A Figura 4.b. apresenta o cenário do fim do jogo. A mensagem final do jogo varia de acordo com a pontuação do jogador. Se a pontuação for igual ou superior a 13 pontos, o jogo fornece um final positivo com os benefícios obtidos através da inspeção de software. Em caso contrário, um final negativo é apresentado onde são relatados alguns dos problemas encontrados em projetos que não utilizam a inspeção de software. InspSoft possui três níveis de desafios: a) primeiro nível: fácil; b) segundo nível: médio; e c) terceiro nível: difícil. A pontuação do participante é calculada da seguinte forma: Cada item de desafio nos níveis do jogo, possuem o valor de 1 ponto, porém, no primeiro nível o jogo possibilita ao jogador acertar todos os desafios. A pontuação do segundo e terceiro nível serão atribuídos somente se o jogador acertar os desafios propostos.

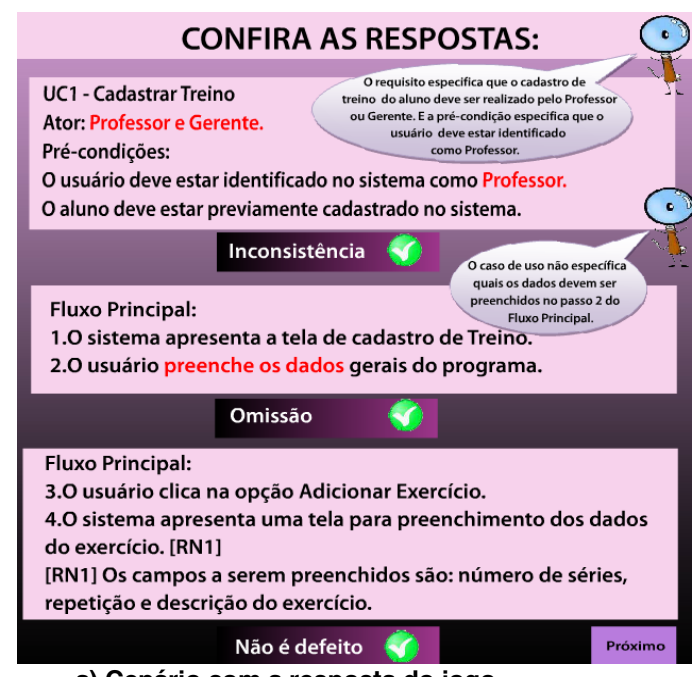

a) Cenário com a resposta do jogo.

\section{Pontuação total da Reunião de Inspeção}

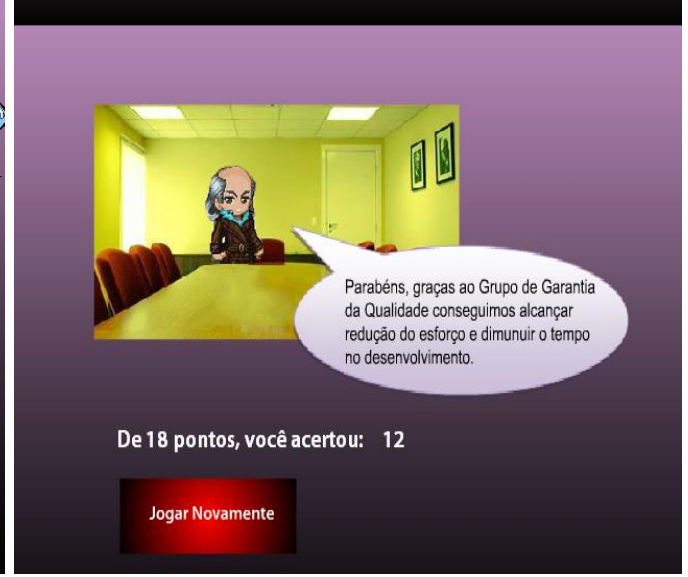

b) Cenário com a pontuação do jogador.

Figura 4. Respostas do Terceiro Nível e Pontuação do jogo. 
Com o objetivo de verificar a efetividade de aprendizagem, motivação e experiência do usuário ao utilizar InspSoft 2.0 foi realizado um estudo experimental, descrito a seguir.

\section{Avaliação do InspSoft 2.0}

Uma avaliação da efetividade dos jogos como forma de aprendizagem se faz necessária para verificar os possíveis benefícios que este tipo de abordagem pode trazer [Wangenheim et al., 2009]. O objetivo do estudo foi estruturalmente definido segundo o paradigma GQM (Goal-Question-Metric) [Basili e Rombach, 1988], segundo a Tabela 3.

Tabela 3. Objetivo do Estudo de Observação segundo o paradigma GQM.

\begin{tabular}{|c|l|}
\hline Analisar & Jogo InspSoft \\
\hline Com o propósito de & Caracterizar \\
\hline Em relação a & Efetividade de Aprendizagem, Motivação e Experiência do Usuário \\
\hline Do ponto de vista & dos Pesquisadores \\
\hline No contexto de & $\begin{array}{l}\text { Ensino de Inspeção de software em uma turma do Instituto de Computação da } \\
\text { UFAM. }\end{array}$ \\
\hline
\end{tabular}

Os participantes do estudo foram 15 alunos da disciplina Qualidade de Software do Curso de Sistemas de Informação do Instituto de Computação da Universidade Federal do Amazonas. Os alunos receberam treinamento sobre inspeção de software, e, após esse treinamento, iniciaram o uso do jogo disponibilizado para o estudo. Os monitores utilizaram formulários de acompanhamento para tomar nota do tempo gasto (com média de 20 minutos), da pontuação obtida e das dificuldades apresentadas. Concluída essa utilização, os participantes preencheram os formulários de avaliação do jogo. Esses formulários foram criados de acordo com o modelo proposto por Savi et al. (2011), descrito a seguir.

\subsection{Modelo de avaliação para jogos educacionais na Engenharia de Software}

Para avaliar o InspSoft 2.0 foi utilizado o modelo de Savi et al (2011). Esse modelo é baseado no modelo de avalição de programas de treinamento de Kirkpatrick (1994), nas estratégias motivacionais do modelo ARCS [Keller, 1987], na área de experiência do usuário e na taxonomia de objetivos educacionais de Bloom [Anderson e Krathwohl, 2001].

Segundo Savi et al. (2011), o modelo possui o foco em jogos que possam ser utilizados como material educacional para apoiar o processo de ensino e aprendizagem da ES, avaliando se o jogo motiva os estudantes a utilizarem a ferramenta como material de apoio, se proporciona experiência positiva com os jogadores e principalmente se o jogo educativo proporciona o conhecimento para o conceito relacionado.

Os itens de avaliação do modelo foram definidos através de estudos de caso, nos quais foram obtidos vinte e sete itens divididos em três subescalas: Motivação, Experiência do Usuário e Aprendizagem. Cada subescala possui uma dimensão com um ou mais itens a serem avaliados. 
O modelo propõe o uso de questionário para avaliar as subescalas, no qual utiliza o formato de resposta para os itens em uma escala que vai de -2 até +2 . Uma nota +1 indica concordância com um item e +2 forte concordância. A nota -1 indica discordância com um item, enquanto -2 forte discordância. Todos os detalhes da documentação para o processo da definição e avaliação estão disponíveis em [Savi et al., 2011].

\subsection{Resultados da Avaliação}

Esta seção apresenta os resultados da avaliação do jogo InspSoft 2.0, com a interpretação dos dados do questionário que está na escala de $-2 \mathrm{a}+2$. Esses dados foram colocados na planilha fornecida pelo modelo de avaliação [Savi et al., 2011]. Quanto maior a quantidade de notas +2 atribuída a um item, maior o nível de concordância dos alunos com a afirmação que está sendo feita sobre o jogo. Assim, é possível identificar os principais pontos positivos e negativos em cada subescala de avaliação.

O gráfico que apresenta os resultados relacionados à Motivação é mostrado na Figura 5. Analisando o gráfico, é possível observar que os alunos se sentiram motivados em todos os itens: Satisfação, Confiança, Relevância e Atenção, de acordo com a frequência de nota +1 ou +2 . Em relação à Satisfação, $74 \%$ alunos estão satisfeitos com a oportunidade de utilizar na prática o conteúdo que aprenderam com o jogo e afirmam que conseguem avançar os níveis no jogo por esforço pessoal. Na avaliação da dimensão de Confiança, $80 \%$ dos alunos concordam que ao passar pelas etapas do jogo sentiram confiança de que estavam aprendendo e $86 \%$ concordaram que o jogo foi fácil de entender.

\section{Motivation}

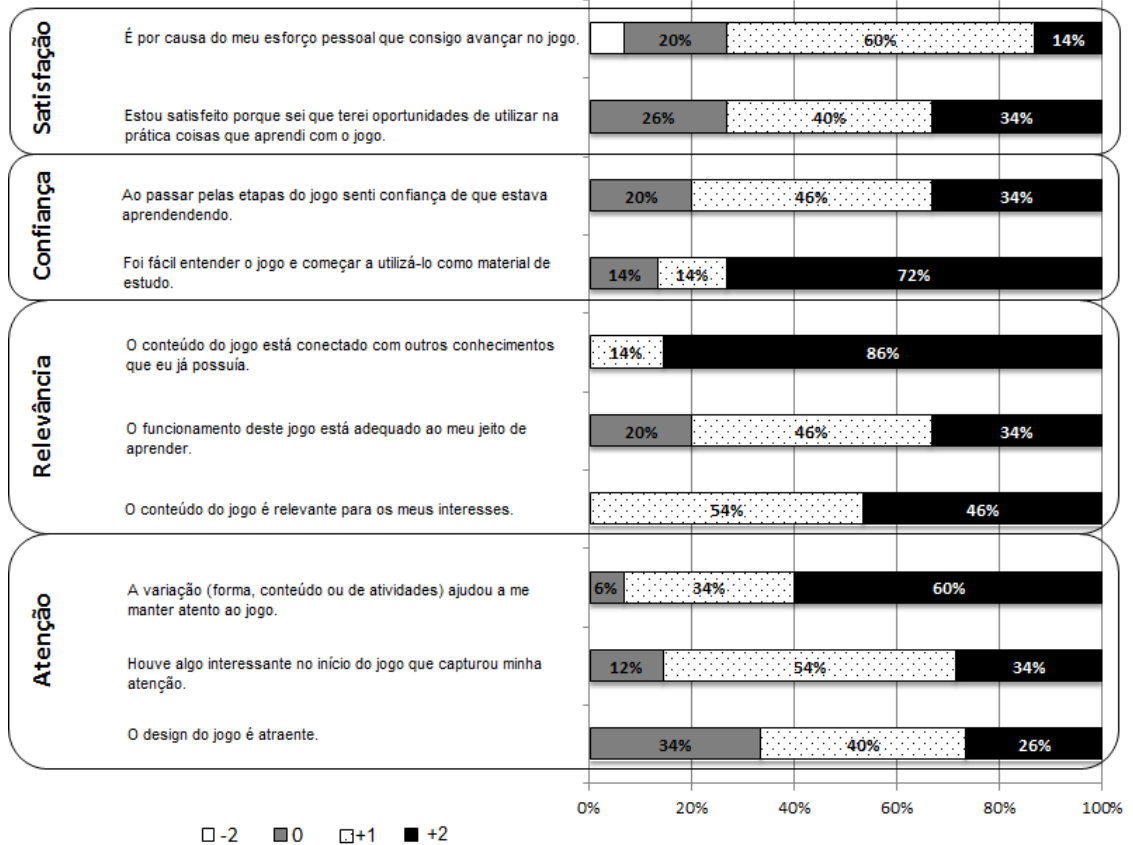

Figura 5. Gráfico de Avaliação da subescala de Motivação. 
A Relevância teve afirmação de $100 \%$ consideram que o conteúdo está conectado com outros conhecimentos e que o jogo é relevante para o interesse dos mesmos Sobre a questão que avalia se o jogo está adequado com o jeito dos alunos aprenderem, $80 \%$ dos alunos concordam com este item. Já em relação à Atenção, $66 \%$ dos alunos consideraram o design do jogo atraente e $74 \%$ avaliaram que a variação do jogo ajudou a mantê-los atentos. Outra questão sobre a Atenção foi à avaliação sobre algo interessante no início do jogo que capturou a atenção dos alunos, onde $88 \%$ dos alunos concordaram com esta questão.

A Figura 6 apresenta os resultados relacionados à Experiência do Usuário. Em relação à Diversão, 94\% dos alunos atingiram os objetivos do jogo por meio de suas habilidades e recomendariam o jogo para seus colegas. Houve concordância com $80 \%$ dos alunos no item que avalia se os alunos conseguiram atingir os objetivos do jogo por meio de suas habilidades. A questão que avalia se os alunos ficaram desapontados com a interrupção e fim do jogo, teve a menor porcentagem na subescala de Experiência do Usuário, com apenas $20 \%$ das notas +1 . Isto pode ser indicação de que o jogo não deveria se estender por mais tempo e sua duração foi adequada.

Sobre a avaliação da dimensão de Competência, 86\% dos alunos tiveram sentimentos positivos de eficiência no jogo e $94 \%$ afirmam que atingiram os objetivos do jogo por meio de suas habilidades. Já em relação ao Desafio, houve uma de concordância de $100 \%$ de que as tarefas do jogo são adequadamente desafiadoras.

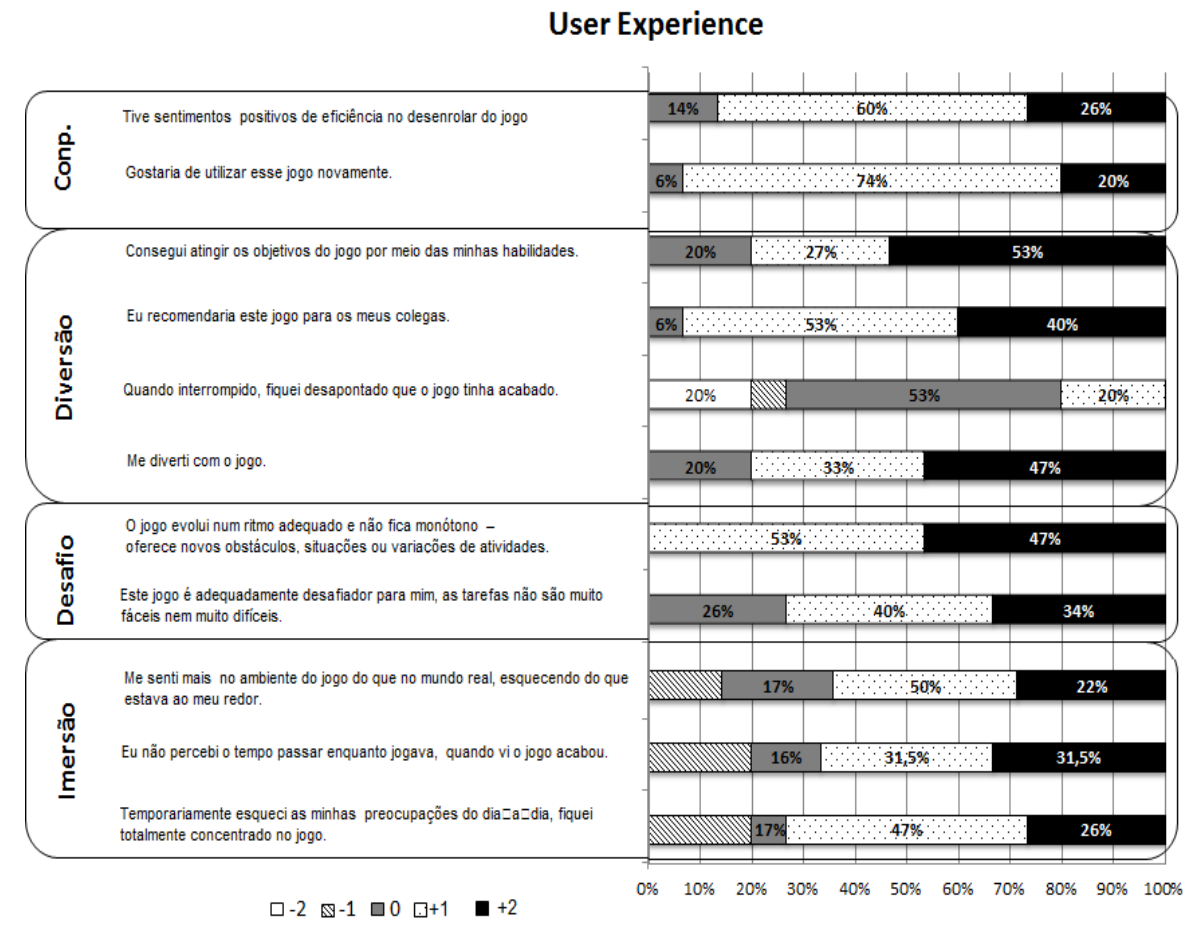

Figura 6. Gráfico de Avaliação da subescala de Experiência do Usuário.

No entanto, o item que pergunta se o jogo não fica monótono e oferece novos obstáculos ou variação de atividades houve concordância com $74 \%$ dos alunos. Sobre a avaliação da dimensão de Imersão nos alunos, $73 \%$ dos alunos afirmam que esqueceram as preocupações enquanto estavam jogando e $63 \%$ ficaram concentrados no jogo. $\mathrm{O}$ 
item "Eu me senti mais no ambiente do jogo do que no mundo real, esquecendo o que estava ao meu redor" teve confirmação de $72 \%$ dos alunos.

Os resultados da subescala de Aprendizagem são mostrados na Figura 7. Nessa subescala é possível observar que o jogo InspSoft 2.0 contribuiu para a aprendizagem da inspeção, ou seja, o jogo ensina a inspecionar. Houve um nível de concordância de $82 \%$ dos alunos afirmando que o jogo traz contribuições para a vida profissional. Dos alunos, $100 \%$ afirmam que o jogo trouxe contribuições na aprendizagem, onde houve $75 \%$ de notas +1 e $25 \%$ de notas +2 para este item. E $100 \%$ dos alunos, consideraram o jogo eficiente em comparação com outras atividades da disciplina com $64 \%$ das notas +1 e $36 \%$ das notas +2 .

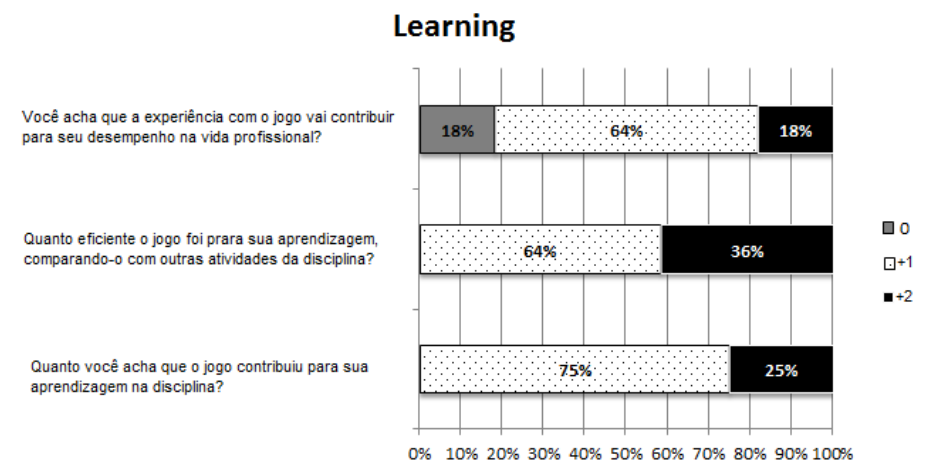

Figura 7. Gráfico de Avaliação da subescala de Aprendizagem.

A Figura 8 apresenta a autoavaliação dos alunos em relação aos objetivos de aprendizagem. Os conceitos avaliados no jogo foram numerados para uma melhor observação: os papéis no processo de inspeção com o Moderador (1), Inspetor (2) e Autor (3), e aos tipos de defeitos encontrados no documento de requisitos como a Omissão (4), Ambiguidade (5), Fato Incorreto (6), Informação Estranha (7) e Inconsistência (8).

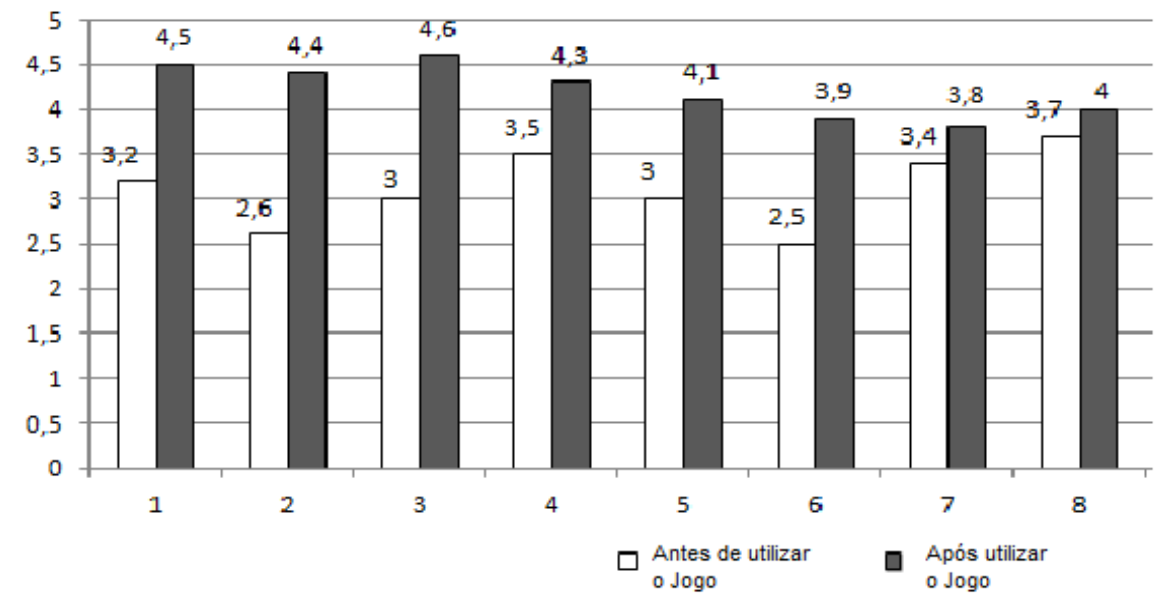

Figura 8. Gráficos dos Objetivos de Aprendizagem.

Ao analisar o gráfico da Figura 8 é possível verificar um aumento no nível de 
conhecimento em todos os objetivos de aprendizagem do jogo. É possível observar que o maior ganho de conhecimento está na parte inicial do jogo, que trata os papéis envolvidos na Inspeção. Estes resultados mostram indícios de que o jogo é um estímulo para os alunos praticarem os conceitos vistos em aulas teóricas. Desta forma, o jogo pode contribuir na melhoria do processo de aprendizagem da inspeção de software, e, por conseguinte, na formação de profissionais mais preparados para inspeções na indústria de software.

\section{Conclusões e Trabalhos Futuros}

O jogo InspSoft é uma alternativa para auxiliar a aprendizagem em inspeção de software de forma lúdica. Neste artigo é apresentada a evolução do jogo, o InspSoft 2.0 que foi realizada através dos resultados de uma avaliação preliminar. O InspSoft utiliza conceitos e exemplos de inspeção advindos da literatura. Além disso, possui três níveis, onde cada nível busca ensinar determinadas partes do processo de Inspeção. No primeiro nível são ensinados os papéis relacionados ao processo de inspeção de Software. No segundo nível, são ensinados conceitos de tipos de defeitos e solicita dos jogadores uma adequada classificação em determinadas situações. Por fim, no terceiro nível, é fornecida uma especificação de um caso de uso para o jogador analisar em relação aos tipos de defeitos.

Com o intuito de verificar a efetividade do jogo foi utilizado o modelo de Savi et al. (2011), um modelo específico para avaliação de jogos educacionais. Os resultados da avaliação foram satisfatórios em relação à primeira versão do jogo [Lopes et al., 2012], no qual obteve uma média de $68 \%$ de notas positivas para os itens de avaliação na subescala de avaliação da Motivação e $55,12 \%$ de notas positivas na subescala de avaliação da Experiência do Usuário. Para a subescala de avaliação de Aprendizagem, houve $67 \%$ das notas positivas.

Os resultados obtidos na avaliação do InspSoft 2.0, para a subescala de avaliação da Motivação houve uma média de $75 \%$ nos itens de avaliação para notas positivas, nas dimensões de Satisfação, Confiança, Relevância e Atenção. Em relação à Aprendizagem, houve uma média de $94 \%$ de notas positivas e na média de autoavaliação dos alunos, em todos os conceitos abordados pelo jogo, os alunos consideraram eficiente para a aprendizagem dos mesmos. Na avaliação da Experiência do Usuário, a dimensão da Imersão recebeu notas negativas em todos os itens de avaliação, com uma média de $14 \%$, sendo uma observação a ser melhorada em um trabalho futuro. Porém, houve uma média de $78,7 \%$ nos itens de avaliação para notas positivas dos itens na subescala nas dimensões de Competência, Desafio, Imersão e Desafio.

Uma limitação da abordagem proposta é em relação à interação com outros jogadores, pois o InspSoft pode ser utilizado apenas por um jogador e as inspeções na indústria são realizadas por uma equipe com papéis específicos do processo de inspeção.

Como trabalhos futuros com o objetivo de tornar o jogo mais personalizável, pretende-se incluir outros produtos de trabalho, tais como: a) estórias do usuário, b) User Interface (UI), c) casos de teste, d) código fonte e e) permissão para interagir com outros jogadores, para que os alunos possam compreender a interação necessária na equipe de inspeção durante a verificação. Desta forma, o jogo poderá ser utilizado no ensino de inspeção de artefatos gerados em diversas etapas do processo de 
desenvolvimento de software, simulando uma inspeção por uma equipe qualificada. Além disso, o jogo InspSoft 2.0 será disponibilizado para o público em geral. Sendo assim, espera-se que a indústria e a academia possam utilizá-lo como ferramenta de apoio ao ensino da inspeção, contribuindo com a qualidade dos produtos de software.

\section{Referências}

Alexander, M., Beatty, J. (2008) "Effective Design and Use of Requirements Engineering Training Games". In: Requirements Engineering Education and Training, REET'08. IEEE, pp. $18-21$.

Anderson, L.W., Krathwohl, D.R., Airasian, P. W, Cruikshank, K.A., Mayer, R. E., Pintrich, P. R., Raths, J., Wittrock, M. C. (2001). "A Taxonomy for Learning, Teaching, and Assessing: a Revision of Bloom's Taxonomy of Educational Objectives". Longman, NewYork, 336p.

Bartié, Alexandre, (2002). "Garantia da Qualidade de Software: Adquirindo Maturidade Organizacional". Rio de Janeiro: Campus, 291p.

Basili, V. R., Rombach, H. D. (1998). "The TAME Project: Towards Improvement - Oriented Software Environments". IEEE Transactions on Software Engineering. v. 14, n. 6, pp. 758773.

Devellis, R. F. (2003) "Scale Development: Theory and Applications", $2^{\text {nd }}$ ed., SAGE, Thousand Oaks, CA, 171p.

Fagan, M.E. (1976). "Design and Code Inspection to Reduce Errors in Program Development", IBM Systems Journal, vol. 15, no. 3, pp. 182-211.

Kalinowski, M., Spinola, R.O., Travassos, G.H., (2004). "Infra-Estrutura Computacional para Apoio ao Processo de Inspeção de Software", III Simpósio Brasileiro de Qualidade de Software, Brasília, Brasil, pp. 62-77.

Keller, J.M., (1987). "Development and Use of the ARCS Model of motivational Design". Journal of Instructional Development, v. 10, n. 3, pp. 2-10.

Kirkpatrick,D. L., (1994). "Evaluating Training Programs - The Four Levels". Berrett-Koehler Publishers, 289p.

Kochanski, D., (2009). "Um framework para apoiar a construção de experimentos na avaliação empírica de jogos educacionais". Dissertação de Mestrado, Programa de Mestrado Acadêmico em Computação Aplicada. Universidade do Vale do Itajaí.

Lopes, A. C., Marques, A. B., Conte, T. U., (2012). "Avaliação do Jogo InspSoft: Um Jogo para o Ensino de Inspeção de Software”. Anais do FEES12 - Fórum de Educação em Engenharia de Software, Natal-RN, Brasil, Setembro 26 e 27, 2012.

Magalhães, A. L. C. C., (2008). “A Importância do Controle da Qualidade na Melhoria de Processos de Software”. In II Workshop de Empresas (W6 - MPS.Br), Campinas, 8p.

Monsalve, E. S., Werneck, V. M. B., Leite, J. C. P., (2010). "SimulES-W: Um Jogo para o Ensino de Engenharia de Software". Anais do FEES 2010/ CBSoft 2010. SBC, 2010. v.1. pp. $17-26$

Pötter, H., Schots, M., (2011). "InspectorX: Um Jogo para o Aprendizado em Inspeção de Software". Anais do FEES 2011 - Fórum de Educação em Engenharia de Software/ CBSoft 2011, São Paulo-SP, Brasil, Setembro 28, 115p. 
Savi, R.; Wangenheim, C., Borgatto, A., (2011). "Um Modelo de Avaliação de Jogos Educacionais na Engenharia de Software". Anais do XXV Simpósio Brasileiro de Engenharia de Software (SBES), São Paulo, pp. 194-203.

Silva, L.F.S., Chapetta, W.A., Travassos, G.H., (2004). "Inspeções de Requisitos de Software Utilizando PBR e Apoio Ferramental". Simpósio Brasileiro de Qualidade de Software (SBQS).

SEI - Software Engineering Institute. (2010). CMMI for Development, Version 1.3, CMMI-DEV v1.3, CMU/SEI 2010-TR-033, Technical Report, Software Engineering Institute.

Sauer, C., Jeffery, D.R., Land, L., Yetton, P., (2000). "The Effectiveness of Software Development Technical Review: A Behaviorally Motivated Program of Research", IEEE Transactions on Software Engineering, 26 (1), January, pp. 1-14.

Softex. (2011). Associação para Promoção da Excelência do Software Brasileiro - Softex MPS.BR - MPS.BR - Guia de Implementação - Parte 3: Fundamentação para Implementação do Nível E do MR-MPS:2011.

Thiry, M., Zoucas, A., Gonçalves, R., Salviano, C., (2010). "Aplicação de Jogos Educativos para Aprendizagem em Melhoria de Processo e Engenharia de Software", In: Anais do VI Workshop Anual do MPS (WAMPS 2010), pp. 118-127.

Travassos, G. H., Shull, F., Carver, J. (2001). "Working with UML: A Software Design Process Based on Inspections for the Unified Modeling Language". Advances in Computer, Vol. 54, pp: 36-99.

Wangenheim, C., Kochanski, D., Savi, R. (2009) "Revisão Sistemática sobre Avaliação de Jogos Voltados para Aprendizagem de Engenharia de Software no Brasil”. In: Anais do FEES 2009/ XXIII SBES, Fortaleza, Brasil, pp. 41-48. 